\title{
Bases łécnico-científicas para apoiar a regulamentação ambiental da aquicultura em águas da União

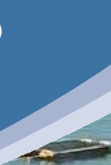

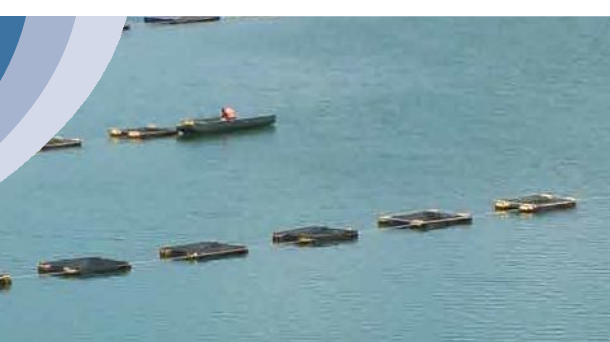

....

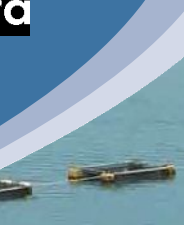
-

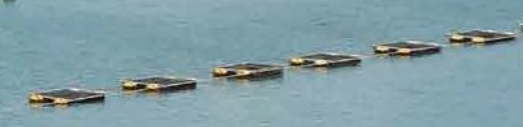
Uniăo

Fernanda Garcia Sampaio', Consuelo Marques da Silva ${ }^{2}$, Daercy Maria Monteiro de Rezende Ayroza ${ }^{3}$, Clóvis Ferreira do Carmo ${ }^{3}$, Daniela Ferraz Bacconi Campeche', Gilberto Caetano Manzoni ${ }^{4}$ e Juliana Lope da Silvas.

'Empresa Brasileira de Pesquisa Agropecuária; ${ }^{2}$ Consultora especialista em monitoramento ambiental; Innstituto de Pesca, Agência Paulista de Tecnologia dos Agronegócios, Secretaria de Agricultura e Abastecimento; "Universidade do Vale do llajai - Escolo ga Mur, Cienra da União da Secretaria Especial da Aquicultura e da Pesca da Secretaria Geral da Presidência da República.

Oesforço de reunir traba hos cientificos realizados em território nacional na temática do monitoramento ambiental da aquicultura resultou em um material impar para subsidiar diferentes aspectos da política de ordenamento da aquicultura em águas da União. Os dados coletados também fornecem base para nortear analistas de meio ambiente nos processos de licenciamento ambiental e produtores na gestão e manejo dos seus empreendimentos. A compi ajcou dos dados científicos é cinco insitr um ento crientado
para embasar a continuidade das pesquisas nesta temática.

Pesquisadores de mais de 40 instituiçöes apresentaram seus resultados compilados em 30 textos. Os dados foram obtidos em projetos já desenvolvidos ou em desenvolvimento, e que em sua maioria foram publicados

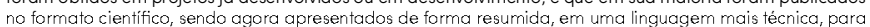
tornar as informações acessiveis ao público e aplicáveis ao monitoramento ambiental da aquicultura.

Os trabalhos estão apresentados em dois capitulos: um de piscicultura em tanque-rede em reservatórios e açudes, e o outro de maricultura, com informaçōes sobre piscicultura, mitilicultura, ostreicultura e algicultura A maior parte dos trah ra. A maior pate dos trabalhos foram realiza refletem a desses trabahos foram realizados por longos periodos, havendo pesquisas executadas há mais de 10 anos. Os capítulos são iniciados com um resumo sobre as principais condicionantes solicitadas nas licenças Os capitulos são iniciados com um resumo sobre as principais condicionantes solicitadas nas licenças
ambientais de parques aquicolas. Nas tabelas apresentadas por Mignani et al. (p. 11 e 63) são compiladas ambientais de parques aquicolas. Nas tabelas apresentadas por Mignani et al. (p. 11 e 63) são compiladas
as variáveis estabelecidas na Resolução CONAMA No 357/2005, na Resolução CONAMA No 413/2009 e alguns parâmetros espećl'icos de Órgäos Estaduais de Meio Ambiente (CEMAs).

\section{ESTRATÉGIAS DE MONITORAMENTO}

Foram apresentadas diversas estratégias de monitoramento ambiental da aquicultura (Figura 1). A maioria dos trabalhos utilizou variáveis de qualidade da água, sendo que alguns pesquisadores aplicaram o Índice de Estodo Trófco (IET), ferramenta já estcbelecida para avaliacâo de impocto no mon'toramento de corpos hídricos. Também foram relatados artigos com avaliação de impactos no sedimento, nos organismos bentônicos, na ictiofauna, na comunidade zooplactônica e com avaliação dos efeitos da sazonalidade e/ou da alteração do volume útil dos reservatórios. Outros estudos envolveram uso de sensoriamento remoto, avaliação da sustentabilidade do sistema produtivo, regularização e ocupação adequada dos empreendimentos, aspectos socicis; autodiagnóstico e influência de variáveis meleorológicas.

Piscicultura em tanque-rede em reservatórios e açudes - a maioria dos dados de monitoramento são provenientes de estudos realizados em criaçōes de tilápia do Nilo (Oreochromis niloticus), com exceção de uma pesquisa realizada na produção de tambaqui (Colossoma macropomum) e outra na de pacu ( $P i a-$ ractus mesopotamicus). Regionalmente, os trabalhos estão concentrados no Sudeste, com dados de São Paulo e Minas Gerais, e no Nordeste com resultados da Bahia, Ceará, Paraiba, Pernambuco e Rio Grande do Norte. Somente dois trabalhos foram executados no Centro Oeste, em Tocantins, e um no Sul no Parana. a) Qualidade da água:

Em relação as estratégias de monitoramento, grande parte das pesquisas compara as áreas de produção de peixes com áreas sem produção (controle). O monitoramento normalmente foi realizado com bas na qualidade da água, utilizando sondas multi-paramétricas, ou coleta de amostras para posterior análise da concentração de nutrientes em laboratório.

Santos el al. (p. 13) nöo verificaram influêncio da piscicul tura em parâmetros como o pH, oxiqêrnio dis-

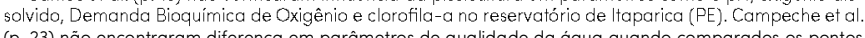
(p. 23) não encontraram diferença em parâmetros de qualidade da água quando comparados os pontos a montante, jusante e no centro da área de produção de uma piscicultura no reservatório de Sobradinho (BA). Sampaio, E. et al. (p. 41) demonstraram que a piscicultura não alterou os parâmetros estabelecidos na Resoluçço CONAMA N $413 / 200$, as variáveis mantiveram-se dentro dos limites definidos na Reso ução CONAMA N $357 / 2005$ e não houve altercçăo do IET, quando ava liadas em áreas sob influência do Parques Aquicolas no reservatório de Três Marias (MG). O mesmo foi encontrado por Matos et al. (p. 31) no monitoramento de piscicultura no reservatório do Lajeado (TO), cujos resultados se enquadraram nas recomendaçoes. Os autores afirmaram que pelo fato do IET se apresentar elevado tanto na área de produçäo como no controle "[...] é possivel afirmar que a aquicul tura näo apresentou impactos significativo no corpo hidrico"

Ayroza et al. (p. 37), que monitoram pisciculturas no estado de São Paulo há mais de dez anos, detectaram aumento no leor de nutrientes em al gumas áreas aquicolas, mas sem o oumento de eutrofizaçăo
caracterizando o "[...] baixo impacto da atividade nos moldes que é praticada atualmente".

Cunha et al. (p. 15) concluiram que a piscicultura monitorada no reservatório de Itaparica (PE) provocou perturbações de baixa intensidade na qualidade da água sem, entretanto, comprometer o ambiente te na capacidade de assimilação das perturbações na qualidade de água provocadas pelo processo de produção atualmente empregado".

Crispim et al. (p. 17), monitorando pisciculturcs no açude do Carne iro (PE), verificaram que ' $[. .$.$] nas pro-$ xida doce de rotíferos, que sco um grupo de an imais que desenvo vem me's em ambientes mais eutrofizados". Os autores que encontraram elevadas concentraçōes de fósforo nas áreas monitoradas, apontaram que os indices elevados também ocorreram nas áreas controle. De maneira que, ou os corpos hídricos já apresentavam elevados teores de fósforo, ou existiam outras fontes que elevaram os niveis de fósforo destes ambientes, além da piscicultura. Também, Benassi et al. (p. 35), no monitoramento ambiental de parques acuicolas no reservatório de taiou, afirmaram aue em uma das áreas monitoradas os tecres ce fósforo ic eram elevados devido às cargas de outras fontes.

Alguns autores questionaram a efetividade do monitoramento ambiental com base nos parâmetros usualmente utilizados. Melo Junior e Dias (p. 21) apontaram, de forma interessante, que a análise da água superficial näo demonstra a realidade que demanda o monitoramento da alividade de piscicultura. O

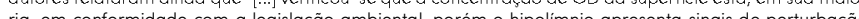
ria, em conformidade "om a legislação ambiental, porém o hipolimnio apresenta sinais de perturbaçăo ve impanco efe retivas se necesśrio. 
Nos textos, destacam-se também o uso das imagens de satélite, permitindo um macro monitoramento dos impactos. No trabalho de Leão et al. (p. 49) foi considerado satisfatório o uso de imagens coletadas pelo sensor OLI, como forma de monitorar os impactos da piscicultura no reservatório de Furnas (MG). A utilização desta ferramenta pode ser expandida para outros reservatórios, sendo considerada um recurso capaz de prop

Grande parte dos trabalhos foram apresentados no semiárido brasileiro e seus resultados indicaram algumas questöes preocupantes para o desenvolvimento sustentável da piscicultura nesta regiäo. Crispim algumas questöes preocupantes para o desenvolvimento sustentável da piscicultura nesta regiäo. Crispim al. (p. I) apontaram a tercencia ce cutrofizaçao destes ambientes, que poce ser potencializada pe

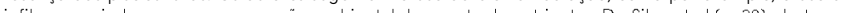

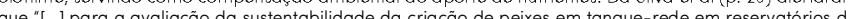
que "... pa icos estäo de par propus priticas publicas para a regulamentches múltiplos usos e das características sazonais destes copos hídricos.

\section{MARICULTURA}

No capítulo da aquicultura marinha, os trabalhos estẫo concentrados em Santa Catarina, sendo em sua maioria, referentes a malacocultura, com apenas um trabalho de algicultura. Existem ainda trabalhos no estado de São Paulo, com ostreicultura, e, fora deste eixo, houve somente um trabalho de piscicultura marinha no Rio de Janeiro e um de algicultura no Ceará.

De maneira geral, os resultados relacionados com a malacocultura comprovaram que os cultivos, no atual estado de desenvolvimento, não estão alterando os parâmetros hidrobiológicos solicitados nas resoluções do CONAMA (357/2005 e 413/2009). Barbieri e Henriques (p. 82) concluíram que o cultivo de ostras em Cananéia [SP] não causcu incremento significativo nas concentraçōes de amônia, ritrito e nitrato, en relação aos valores estabelecidos pela Resolução CONAMA N 357/2005 para águas salobras da Classe alimento, uma vez que são atividades produtivas que não utilizam insumos como ração.

Estudo conduzido por Mignani et al. (p. 76) concluiu que os altos indices de coliformes fecais termoolerantes possivelmente estão associados ao aumento do turismo na região e às águas carreadas por olerantes possivelmente estáo associados ao aumento do turismo na regiáo e as aguas carreadas por mo fazendas marinhas servem como um bom indicador dos niveis de poluição fecal que atingem essas áreas, em SC principalmente aquela gerada pelas cidades. Porém, esse parâmetro não serve como indicador dos impactos a mbientais causados pelo cultivo de moluscos. Coliformes são bactérias que indicam a presença de fezes de animais de sangue quente, que não é o caso dos bivalves".

O monitoramento de coliformes termotolerantes, em especial E. coli, integram os parâmetros elencados tanto na Resolução CONAMA No 413/2009 como na Resoluçäo CONAMA No 357/2005 (Mignani et al. (p.64)). Contudo, é importante destacar que esse parâmetro deve ser monitorado nos moluscos e nas áreas de cultivo, năo para avaliaçäo de impacto, mas para certificação dos moluscos cultivados. De acordo om a Instrução Normativa 7/2012, este parâmetro em conjunto com biotoxinas originárias de microalgas estabelecem critérios de retirada dos moluscos das áreas de cultivo para o consumo humano.

A abordagem multidisciplinar apresentada por Manzoni et al. (p. 72) possui uma série de resultados de monitoramento do cultivo de moluscos marinhos na Enseada da Armação do Itapocorói (SC). Foram considerados alem dos parametros exigidos nas resoluçôes CONAMA, outros que forneceram indicaçōes da interface sedimento-água, depósito da maior parte dos resíduos originários do cultivo de moluscos, que segundo os autores merecem uma atenção especial.

Parc a monitoramento de parâmetros físico-químicos e clorofila-a na água não foram verificcdos irrpaclos comparando cam areas controle. Omateria par culado em suspensco, sob influencia do callivor da sazonalidade e hidrodinâmica local, produziu um aum estudos apresentados por Manzoni et al. (p. 72) indica que os cultivos estáo de fato contribuindo com axa de sedimas os maiores valores foram observados próximos a undo. Resultados demonstram que "[...] apesar das estruturas e os organismos cultivados estarem contribuindo na taxa de sedimentação isso parece não interferir na concentração dos nutrientes dissolvidos na água. Mesmo os parâmetros monitorados estando dentro dos limites estabelecidos pela Resolução 357 , os cultivos parecem contribuir na alteração da qualidade da água da Enseada, porém de maneira gradual e enta". O grupo de pesquisadores reforça a necessidade de avaliar além dos parâmetros na coluna d'agua locais com e sem cultivos de moluscos.

Landuci et al. (p. 66) apontaram para efeitos mínimos ambientais na qualidade da água na área de produção de bijupirá (Rachycentron canadum) no litoral Sul do Rio de Janeiro quando comparados os produção de bijupirá (Rachycentron canadum) no litoral Sul do Rio de Janeiro quando comparados os
dados obtidos com o estabelecido na Resolução CONAMA N ${ }^{\circ} 357 / 2005$ ressaltando que os impactos foram maiores quando utilizados alimentos úmidos em comparação ao extrusado.

Outro grupo importante de resultados refere-se às pesquisas com algas. Hayashi e Santos (p. 86) sugeriram critérios para o monitoramento ambiental da espécie Kappaphycus alvarezii, de forma a garantic um cultivo seguro da espécie exótica no litoral brasileiro. São destaques do trabalho o diagnóstico do baix risco de estabelecimento da espécie em Santa Catarina e a sugestão de "[...] propor um protocolo de monitoramento ambiental a ser realizado pelo produtor que desejar cultivar alga, que seja, ao mesmo tempo, activel de ser conduzido por eles e que atenda, minimamente, as exigências dos órgãos ambientais".

Além disso, Valenti et al. (p. 84) apresentaram resultados preliminares de um estudo que avaliou a taxa de sedimentação de materiais em suspensão e concentração de nutrientes em cultivo experimental da Hypnea pseudomusciformis na praia de Flecheira (CE). Os autores concluiram que "[...] o cultivo de macroalgas poce ser consicerado uma alividade que nco alera sign fícativarmente a qualidade co água resescala de produção. O grupo destaca ainda que a área estudada apresentou concentração de nutrientes acima do li mite estabelecidos para a Classe 1, porém "[...] para fine de licenciamento, é im portante que haja consideração das particularidades do corpo hídrico em que o empreendimento aquicola será instalado, para que o mesmo náo modi que o ambiente a ponto de afe tar suc resiliêncic. Se for con siderado o pcâmetro da Resoluçãoo, não seria possivel qualquer atividade aquicola marinha naquela região. Portanto, há o desafio de se odequar a regulamentaçäo paro licenciamento ambiental de atividades aquicola
marinhas no Brasil".

\section{CONSIDERAÇÕES GERAIS}

Trabalhos destacam claramente a problemática da instalação da piscicultura em pequenos açudes no semiárida, da influêncía da diminuiçäo do volume útil dos reservatórios no impacto da ativicade e no semiárido, da influência da diminuiç̧a do vo ume útil dos reservalórios no impacio da alivicade e substanciais.

Dentre o cori unto de desa fios cpontados para o monitoramento da aquicultura está a necessidade de - avaliar o real impacto da atividade, estimulando o desenvolvimento de ferramentas específicas paro esse fim, que permitam o alcance ce resultados que apoiem a acosco de práticas de maneio evitanco ou mpactos gerados.

Apesar do grande esforço dos grupos de pesquisa em atuar no tema, percebe-se a necessidade de ma maior integração entre as instituições e de que os resultados sejam apresentados de forma a sere fetivamente utilizados, visando o desenvolvimento sustentável da aquicultura.

A maioria dos trabalhos não utiliza os procedimentos do Programa de Monitoramento Ambiental que constam no Anexo VI da Resolução CONAMA No 413/2009, porém, fazem uso desse instrumento e da Resolução CONAMA N 357/2005, como base para análise e discussão dos dados obtidos. É interessante observar que a maior parte dos resultados mostram não haver impactos da atividade aquicola (continenta emarinha), demonstrando a necessidade de uma ampla discussáo quanto a efetividade do uso desses instrumentos juridicos para o acompanhamento dos impactos da atividade. Desta forma, podemos inferir tanto que os instrumentos náo tem sido eletivos para orientar modelos de monitoramento quanto podem estar gerando maior ônus para os produtores em monitorar algo não efetivo. Neste sentido Melo Júnior e Dias (p. 21) sugerem que "[...] A piscicultura em tanque-rede necessita de métodos de monitoramento limno óg co que sejam simp ese eficientes".

Por outro lado, nas discussões também se deve considerar que os sistemas de cultivos atualmente realizados no Brasil, nöo tem provocado impactos significativos, por náo terem atingido a capacidade su porte dos ambientes onde estão alocados, em decorrência das espécies e biomassa cultivada, ou ainda, da hidrodinâmica que promove a dispersão dos residuos gerados. E que considerando que todo cessionário de área aquicola, recebe uma autorização para produzir uma determinada quantidade de pescado e essa produção está de acordo com um cálculo prévio de capacidade de suporte (Torigoi et al.; p. 59).

Vale a pena destacar a complexidade e os custos que envolvem a logistica e análises dos parâmetros de qua idade do monitoramento preconizado nas Resoluç̧̈es COVAMA No 413/2009 e 357/2005 e a difimbiental deve ser uma ferramenta para assegurar que um empreendimento não esteja causando impacto, ou, caso cause, seja 
possivel mitigá-lo. A inclusão de variáveis pouco efetivas, pelas OEMAs, nos processos de licenciamento ambiental não contribui para a redução dos impactos e gestão da atividade. Sampaio, F. et al (p. 45) apontaram que "i... O monitoramento preconizado nos processón

A Resolução CONAMA N 413/2009 estabelece no Programa de Monitoramento Ambiental, que o cessonário deve apresentar um paro de monitoramento da água e efluentes e um Re atório Técnico com uma analise dos parametros hidrobiologicos, de acordo com a requência estabelecida pelo orgáo ambienta. constando as principais al feraços decor, antes do enp a res. Portanto, novamente fica evicente c impotância do m

Os aquicultores são os principais interessados na manutenção da qualidade da água e dos padrões ambientais do local onde estão produzindo, devido a influencia desses fatores nos índices produtivos e no sucesso economico da produção. Uma vez que os parâmetros exigidos nos mecanismos legais demons tram não serem efelivos para assegurar um desenvolvimento mais justo e a geração de informações para a mitigação de potenciais impactos, há necessidade do estabelecimento de novas ferramentas para 0 monitoramento. Alguns grupos de pesquisa já estão propondo novos caminhos, como o uso de variáveis

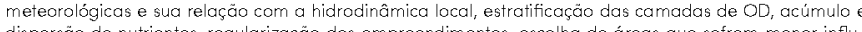
dispersão de nutrentes, regu arizaçco dos empreendimentos, escolha de árecs que sofrem menor influêncla das fiç claramente ações que requerem a execução direta de órgãos de pesquisa e extensão ou de gestão de recursos hidricos.

Sampaio, F. et al (p. 45) ircicam como desa fios a necessidade de '[...] rever os parâmetros sugeridos na Resolução CONAMA N $413 / 2009$, para que esta seja mais efetiva na proposiçáo de parâmetros para - monitoramento ambiental da aquicultura e a possibilidade de mitiga-los quando houver. Discutir juntos aos órgãos estaduais de meio ambiente e gestores de recursos hidricos as questöes relacionadas a monitoramento antiental da acuicultura, e a baixa eficiéncic do monitoramento com base somente no qualidade de água:"

A síntese dos dados indica que o monitoramento sedimentológico tem maior representatividade para subsidiar o monitoramento da aquicultura (Figura 2).

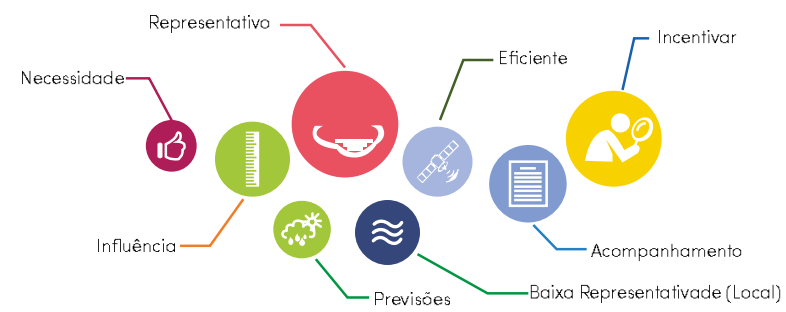

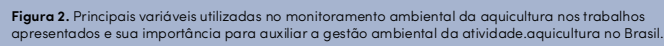

Ainda, grande parte dos autores apontou a necessidade de maiores discussões quanto as metodologias adotadas no monitoramento ambiental da aquicultura, de padronização dos métodos de coleta e análise e da publicitaçáo das informaçoes. Houve a sugestáo de um trabalho em rede, envolvendo piscicultores drográficas.

No II Workshop da Rede de Monitoramento, realizado em abril de 2018, foi apresentada pela EMBRAPA/ SEAP uma proposta de um Plano Nacional de Monitoramento Ambiental da Aquicultura em Águas da
União (PMA; Sampaio et al., 2019). O documento concentra os esforços de monitoramento no sedimento do local do empreendimento para o diagnóstico quanto ao nivel de impacto da atividade de aquicultura no ecossistema aquático. O compartimento ambiental tem uma elevada capacidade de incorporação de compostos orgânicos e inorgânicos e comumente apresenta baixa taxa de liberação dos mesmos, possibiltando até a identificaçăo da fonte de despejo do po vente. A proposta pretende agregar as açoses dos cessionários das áreas de produção e das instituiçoes de pescuisa, de fisca izaçâo e ordenamento, de outorga e operaço em un único $\mathrm{P}$ cro. Dessc forma, unificar cs diversas ccés de monitoramento ac

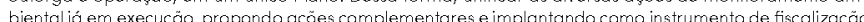
- Relatório de Produçấo Anual (RPA), de modo auto-declaratório, em conjunto com o acompanhamento dos dados de qualidade do sedimento lacustre.

Nos orientaçces do FMA (Figura 3), os sistemas produlivos foram classificados em pequenos, médios e grandes, sendo que por analogia equiparam-se como Disoensado, Licenciomento Simo lificado e Licencicgrandes, sendo que

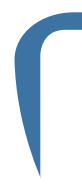

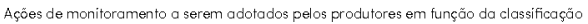
dos empreendimentos com base no volume de produção (t/ano).

\begin{tabular}{|c|c|c|c|}
\hline Classificação & Nivel (t/ano) & Ação & Frequência \\
\hline Pequenos & até 50 & Envio do Relatório de Produção Anual & Anual \\
\hline \multirow[t]{2}{*}{ Médios } & \multirow[t]{2}{*}{$>50$ até 500} & Envio do Relatório de Produção Anual & Anual \\
\hline & & Análise de sedimento & Anual \\
\hline \multirow[t]{4}{*}{ Grandes } & \multirow[t]{4}{*}{$>500$} & Envio do Relatório de Produção Anual & Anual \\
\hline & & Análise de sedimento & \multirow[t]{2}{*}{ Anual } \\
\hline & & $\begin{array}{l}\text { Apresentação do acompanhamento da qualidade da } \\
\text { água pelo empreendimento }\end{array}$ & \\
\hline & & $\begin{array}{l}\text { Plano de Monitoramento e Gestão Ambiental (PMGA) } \\
\text { com açōes de controle de migração }\end{array}$ & Anual \\
\hline
\end{tabular}

Figura 3. Resumo da proposta de monitoramento do Plano Nacional de
Monitoramento Ambiental da Aquicultura em Aguas da Uniāo (PMA).

Como a presente publicação é parte das ações da Rede Nacional de Pesquisa e Monitoramento Ambiental da Aquicultura em Águas da União (Rede), os encaminhamentos aqui apresentados serão a base da Rede nos proximos anos. Esta primeira etapa demonstrou o quanto avançamos nas ferramentas de monitoramento e gestão ambiental da aquicultura. As informaçoes tecnicas apresentadas devem subsidiar as discussōes das principais lacunas que temos hoje na gestão ambiental da aquicultura, ou seja, o rea impacto desta atividade no meio ambiente e o efetivo monitoramento para a gestão da atividade. Esta publicação será um valioso instrumento para subsidiar o processo de licenciamento ambiental da aquicultur com base en dados científicos nocionais.

A consolidação do presente trabolho permitiu verificar oue as açoes de monitoramento ambiental do aquicultura ja são realizadas em grande parte do Brasil e que tem apoio das instituiçōes de pesquisa. Também subsidiou a Rede em uma ampla discussao sobre questôes de ordenamento sustentável da aquicultura em águas da União. Porém, o principal propósito desta iniciativa foi trazer à tona os modelos atualmente adotados no processo de monitoramento ambiental da atividade e discutir junto aos autores da Rede qual caminho devemos seguir.

REFERÊECIAS BIBLIOGRAFICAS:

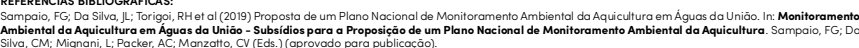

